\title{
Percepção dos pais e responsáveis sobre o uso do canabidiol em crianças e adolescentes com epilepsia
}

\author{
Perception of parents and guardians on the use of canabidiol in children and adolescents \\ with epilepsy
}

Percepción de padres y tutores sobre el uso de cannabidiol en niños y adolescentes con epilepsia

Claudirene Milagres Araújo ${ }^{1 *}$, Flávia Andrade Almeida ${ }^{1}$, Maria Luísa do Espírito Santo Del Rio, Priscila Aparecida Ferreira da Silva ${ }^{1}$, Brisa Emanuelle Silva Ferreira'.

\section{RESUMO}

Objetivo: Compreender a percepção dos pais ou responsáveis sobre o uso do Canabidiol em crianças e adolescentes com epilepsia. Métodos: Trata-se de pesquisa exploratória, descritiva, com abordagem qualitativa, onde os sujeitos foram captados pela estratégia "bola de neve" através do WhatsApp e responderam a entrevista pelo Google Forms por meio de questionário semiestruturado. Para avaliar os conteúdos dos depoimentos foi realizada análise temática proposta por Bardin L (2011). Resultados: Participaram da pesquisa 16 pais ou responsáveis. Segundo os sujeitos entrevistados, o Canabidiol pode atuar na melhora cognitiva, motora e melhor interação social o que proporciona melhora na qualidade de vida. Relatam também, alguns entraves na aquisição da substância como alto custo, dificuldades na importação e desconhecimento médico. Conclusão: Foi possível ter a percepção através do relato dos pais sobre a efetiva melhora através do uso do Canabidiol, mas o acesso ao medicamento ainda é um entrave. É de suma importância que o profissional enfermeiro se atualize em relação as possibilidades terapêuticas existentes para trabalhar junto aos pacientes e equipe multidisciplinar nos diversos níveis de atenção primário, secundário e terciário. Desta forma estudos com rigor metodológico devem ser conduzidos para apoiar a eficácia da utilização, verificar efeitos adversos, comprovando sua segurança.

Palavras-chave: Epilepsia, Canabidiol, Epilepsia resistente a medicamentos, Agência Nacional de Vigilância Sanitária.

\begin{abstract}
Objective: To understand the perception of parents or guardians about the use of Cannabidiol in children and adolescents with epilepsy. Methods: This is exploratory, descriptive research with a qualitative approach, where the subjects were captured by the "snowball" strategy through WhatsApp and answered the interview through Google Forms through a semi-structured questionnaire. To evaluate the contents of the statements, a thematic analysis proposed by Bardin L (2011) was carried out. Results: 16 parents or guardians participated in the research. According to the interviewed subjects, Cannabidiol can act in cognitive and motor improvement and better social interaction, which provides an improvement in the quality of life. They also report some obstacles in the acquisition of the substance, such as high cost, difficulties in importing and lack of medical knowledge. Conclusion: It was possible to have the perception through the parents' report about the effective improvement through the use of Cannabidiol, but access to the drug is still an obstacle. It is extremely important that the professional nurse is updated in relation to the existing therapeutic possibilities to work with patients and the multidisciplinary team at the different levels of primary, secondary and tertiary care. Thus, studies with methodological rigor must be conducted to support the effectiveness of use, verify adverse effects, proving its safety.
\end{abstract}

Keywords: Epilepsy, Cannabidiol, Drug-resistant epilepsy, National Health Surveillance Agency.

${ }^{1}$ Centro Universitário de Belo Horizonte (UNIBH), Belo Horizonte - MG.

*E-mail: claudirene_milagres@hotmail.com

SUBMETIDO EM: 12/2021

ACEITO EM: 1/2022

PUBLICADO EM: 2/2022 


\section{RESUMEN}

Objetivo: Conocer la percepción de los padres o tutores sobre el uso de Cannabidiol en niños y adolescentes con epilepsia. Métodos: Se trata de una investigación exploratoria, descriptiva con enfoque cualitativo, donde los sujetos fueron captados por la estrategia "bola de nieve" a través de WhatsApp y respondieron la entrevista a través de Google Forms a través de un cuestionario semiestructurado. Para evaluar el contenido de los enunciados se realizó un análisis temático propuesto por Bardin L (2011). Resultados: 16 padres o tutores participaron de la investigación. Según los sujetos entrevistados, el Cannabidiol puede actuar en la mejora cognitiva y motora y en una mejor interacción social, lo que proporciona una mejora en la calidad de vida. También relatan algunos obstáculos en la adquisición de la sustancia, como alto costo, dificultades en la importación y falta de conocimiento médico. Conclusión: Fue posible tener la percepción a través del relato de los padres sobre la mejoría efectiva a través del uso de Cannabidiol, pero el acceso al fármaco sigue siendo un obstáculo. Es de suma importancia que el profesional de enfermería se actualice en relación a las posibilidades terapéuticas existentes para trabajar con los pacientes y el equipo multidisciplinario en los diferentes niveles de atención primaria, secundaria y terciaria. Por lo tanto, se deben realizar estudios con rigor metodológico para respaldar la eficacia del uso, verificar los efectos adversos y probar su seguridad.

Palabras clave: Epilepsia, Cannabidiol, Epilepsia farmacorresistente, Agencia Nacional de Vigilancia Sanitaria.

\section{INTRODUÇÃO}

A epilepsia é uma doença de hiperexcitabilidade crônica, que se origina de vários defeitos em redes neuronais no cérebro que levam a convulsões recorrentes. Caracteriza-se por descargas elétricas anormais que podem se originar de uma variedade de regiões cerebrais e podendo causar alterações nos comportamentos, consciência e sensações (REDDY DS e GOLUB VM, 2016).

A epilepsia afeta cerca de 65 milhões de pessoas em todo o mundo, com uma incidência de 20 a 70 novos casos por 10.000 pessoas por ano (REKTOR L, et al., 2015). As crianças são as mais afetadas, com prevalência de aproximadamente 5:1000, sendo a faixa etária mais frequente a de zero a cinco anos de idade. Isto pode ser entendido ao considerar que o sistema nervoso das crianças ainda está imaturo, com poucas reações inibitórias das sinapses (FONSECA GS, et al., 2016).

As crises epilépticas podem ser classificadas como crise parcial simples, complexa, secundária generalizada e generalizada. Para diagnosticar como epilepsia é necessário que haja duas ou mais crises espontaneamente, e ao contrário de muitas crenças, a epilepsia não é contagiosa (MINISTÉRIO DA SAÚDE, 2018a).

Segundo a Organização Mundial de Saúde (OMS) o tratamento é realizado de acordo com o tipo de crise apresentada, sendo o Ácido Valpróico o mais utilizado em crises inicialmente generalizadas e a Carbamazepina, Fenitoína ou Ácido Valpróico mais comuns no tratamento de crises de início focal (MINISTÉRIO DA SAÚDE, 2018b).

Há uma necessidade crescente de novas terapias que proporcionem controle efetivo da epilepsia resistente a medicamentos ou refratária e não interfiram na função normal. Recentemente, os canabinoides têm sido sugeridos como potenciais alternativas terapêuticas para alguns pacientes com crises refratárias. Estudos emocionais experimentais e experimentais sugerem que o Cannabidiol (CBD), um constituinte não psicoativo encontrado dentro da planta de Cannabis sativa, pode atuar como um agente antiepiléptico (REDDY DS e GOLUB VM, 2016).

A planta Cannabis sativa é usada para fins medicinais há anos. Múltiplas culturas, já são adeptas ao uso dessa planta. Porém hoje em dia também é sabido seus efeitos adversos. Há indicações do uso dessa planta iniciou-se na China antes da Era Cristã. Uma de suas múltiplas indicações era para tratamento de condições clinicas como constipação intestinal, dores, malária, expectoração, epilepsia, tuberculose, entre outras. $\mathrm{Na}$ Índia seu uso vem sendo feito há 1.000 anos antes de cristo como tratamento de ansiedade, mania e histeria intestinal, dores, malária (OLIVEIRA MT e PAIM RSP, 2015). 
O uso medicinal da maconha se constitui pela ultilização de dois canabinóides primários isolados da planta Cannabis Sativa, e pode ser um tratamento alternativo para epilepsia intratável e resistente ao tratamento, resultando em melhora da função comportamental e cognitiva e redução no número e intensidade das crises. Brucki SM (2015) relata que apesar do reconhecido efeito antiepiléptico do CBD, seu mecanismo de ação, segurança a longo prazo, propriedades farmacocinéticas e interações com outros fármacos, ainda são obscuros. Os pacientes que fazem uso desta substância em sua grande maioria possuem síndromes epilépticas heterogêneas que não responderam a qualquer outro fármaco, ou tiveram sérios efeitos colaterais com os medicamentos disponíveis no mercado.

No Brasil pacientes epilépticos usuários de CBD têm dificuldade com a prescrição e liberação da substância, sendo que em alguns países como o Estados Unidos, a substância é vendida em supermercado (GURGEL HLC, et al., 2019). O primeiro relato de liberação da substância no Brasil foi em 2014, através de uma ação judicial, na $3^{\underline{a}}$ Vara Federal de Brasília, o que permitiu a uma família importar a substância. Após isso, o Conselho Federal de Medicina (CFM) (2014) autorizou os médicos a prescreverem o CBD para pacientes que já tentaram todos os outros medicamentos e não obtiveram melhora.

Em 2014 a Agência Nacional de Vigilância Sanitária (ANVISA) aprovou, por unanimidade, a reclassificação do CBD como medicamento de uso controlado e não mais como substância proibida, não sendo mais necessário ordem judicial para seu uso. O familiar responsável deve conseguir receita da substância e laudo com o neurologista que acompanha a criança, fazer uma declaração de responsabilidade e cadastrar o paciente (ANVISA, 2018).

Desta forma, esse estudo teve como objetivo compreender a percepção dos pais ou responsáveis sobre o uso do canabidiol em crianças e adolescentes com epilepsia.

\section{MÉTODOS}

Foi realizada uma pesquisa exploratória, descritiva com abordagem qualitativa, que fizesse emergir a percepção dos pais ou responsáveis sobre o uso do CBD em crianças e adolescentes com epilepsia. A abordagem qualitativa se preocupa, nas ciências sociais, com um nível de realidade que não pode ser quantificado (MINAYO MC, 2015).

A pesquisa foi realizada através de um roteiro de entrevista estruturado online no Google Forms, disponibilizado através de uma página da internet utilizando a ferramenta Google Drive para a criação do formulário.

De acordo com Gil AC (2010) o instrumento de coleta de dados é importante para garantir a profundidade necessária ao estudo e a inserção do caso em seu contexto, bem como para conferir maior credibilidade aos resultados.

O estudo não foi vinculado a nenhuma instituição, os sujeitos foram os pais ou responsáveis pelas crianças e adolescentes com diagnóstico de epilepsia em uso de CBD, que se dispuseram a participar do estudo. Foi utilizada a técnica "Bola de Neve", para captar os sujeitos, onde o entrevistado que participou do estudo, indicou um novo participante, enviando o número de WhatsApp para os pesquisadores e estas enviavam o link para o provável participante.

De acordo com Vinuto JA (2014), a técnica Bola de Neve é útil para estudo realizado com determinado grupo cujo acesso seja difícil, uma vez que se utiliza de redes de referência, ou seja, contatos. Esta técnica apresenta-se como um processo contínuo de coleta de informações com um conjunto de contatos potenciais, e que pode ser finalizado com base no critério da saturação dos dados.

Aspectos éticos que merecem destaque neste estudo são a garantia de que não houve discriminação na seleção dos participantes, os riscos que envolveram os sujeitos foram mínimos, foi garantido o anonimato e confidencialidade dos entrevistados. O Termo de Consentimento Livre e Esclarecido (TCLE) obedeceu às normas preconizadas na Resolução 466/2012 (CONSELHO NACIONAL DE SAÚDE (CNS), 2012).

Os critérios de inclusão para o estudo foram pais ou responsáveis pelas crianças e adolescentes com diagnóstico de crise epiléptica de difícil controle em uso de CBD e como critérios de exclusão foi garantido o direito de desistência da participação no estudo durante e após o preenchimento do roteiro semiestruturado. 
Após a elaboração do roteiro semiestruturado, para que ele fosse validado, foi enviada a pesquisa por WhatsApp para dois pais ou responsáveis, e após a aplicação foram realizadas as correções e ajustes no roteiro, para que os dados obtidos fossem satisfatórios para o objetivo do estudo. Estes dois sujeitos que participaram da validação prévia não fizeram parte da amostra estudada.

O estudo seguiu orientações expressas na Resolução no 466/2012 do Ministério da Saúde (MS, 2012), que trata de pesquisas com seres humanos e foi submetido ao Comitê de Ética em Pesquisa (CEP) do Centro Universitário de Belo Horizonte (UniBH).

Após aprovação do CEP UniBH sob o CAAE: 10203119.8.0000.5093 e parecer número: 3.021.269 foi enviando aos participantes o convite da participação na pesquisa, através do WhatsApp. Após o entrevistado concordar em participar era enviado o link com o endereço da página que continha o TCLE e o roteiro de entrevista. Quando o entrevistado acessava o link, o TCLE abria para ele ler e dar o consentimento ou não. Após concordar em participar da pesquisa, o participante tinha acesso ao roteiro de entrevista. Ao final do roteiro o entrevistado tinha a opção de deixar um número de WhatsApp indicando um novo participante. Os entrevistados preencheram os roteiros apenas com suas iniciais para garantir o sigilo da coleta de dados.

Foi utilizada uma amostragem por saturação, uma ferramenta conceitual que pode ser empregada em investigações qualitativas (MINAYO MCS, 2015). Para garantir a saturação dos dados, o próximo questionário era enviado quando o anterior já tivesse sido lido e inserido na planilha de resultados feita no Excel para garantir a saturação dos dados.

A Análise de Conteúdo de Bardin foi o referencial utilizado para avaliar os conteúdos que emergiram nos depoimentos. Trata-se de um conjunto de técnicas que decompõem as comunicações, que utiliza procedimentos sistemáticos e objetivos para descrever o conteúdo das informações. Procura conhecer aquilo que está por trás das palavras, sobre as quais se debruça (BARDIN L, 2011). E, por fim, os resultados foram comparados com a literatura científica, a partir de sua síntese.

Para manter o anonimato dos participantes do estudo, as entrevistas foram identificadas por códigos, seguidas do número da entrevista e foram utilizados códigos de identificação E1, E2, [...], para apresentação das falas dos participantes.

\section{RESULTADOS}

Os participantes desta pesquisa foram 16 pais ou responsáveis de crianças ou adolescentes, as quais tinham idade entre 1 e 16 anos. Em relação a renda familiar dos entrevistados obteve-se que $12,5 \%$ têm renda familiar de um a dois salários mínimos, $18,8 \%$ de três a quatro salários mínimos e $56,3 \%$ de cinco salários mínimos ou mais. Quanto à escolaridade dos pais ou responsáveis, $62,5 \%$ possuíam ensino superior completo, $25 \%$ ensino médio completo e $12,5 \%$ ensino fundamental completo.

Os depoimentos foram organizados em três categorias: "Percebendo o CBD como recurso terapêutico"; "Relação sociedade e família com o uso do CBD"; "Entraves na aquisição do CBD".

\section{Percebendo o CBD como recurso terapêutico}

Ao serem questionados alguns pais ou responsáveis, referem uma boa experiência com o uso do CBD em suas crianças e/ou adolescentes com epilepsia. Dentre os benefícios relatam diminuição das crises epilépticas, melhora cognitiva, equilíbrio, fala, visão e autonomia:

“(...) os benefícios foram logo percebidos. Em conjunto com as demais medicações administradas, as crises foram praticamente controladas, a atenção, concentração, $e$ equilíbrio corporal, apresentaram melhora extremamente significativa. Aos poucos, as crises foram reduzindo consideravelmente" (E1).

“(...) minha filha está em uso do CBD há 57 dias, com 20 dias de uso as convulsões cessaram e com 33 dias consegui tirar meio comprimido de Vigabatrina sem prejuízo para ela. Isso foi um avanço devido ter de 30 a 40 convulsões diárias (...)" (E15). 
"Houve uma diminuição das crises convulsivas e melhorou muito o cognitivo dele. Antes do CBD ele não demostrava sentimento de dor, alegria ou tristeza, após o uso do óleo se expressa melhor, consegui descobrir quando ele tem dor ou precisa de algo" (E16).

Outros depoimentos apontam benefícios do CBD em relação à melhora do cognitivo, coordenação, redução da medicação anticonvulsivante e de seus efeitos colaterais e interação com o mundo. Entretanto, estes pais ou responsáveis não relataram redução das crises epilépticas com o uso da substância:

"Iniciamos o CBD com o intuito de controlar as crises convulsivas, depois de tentar diversos medicamentos, em altas doses, com muitos efeitos colaterais. $O$ CBD não foi efeito para eliminar as crises, mas trouxe outros ganhos, como a possibilidade de diminuir as demais medicações, a diminuição significativa dos efeitos colaterais, $o$ humor, a conexão com o mundo, o controle mastigatório etc." (E4).

"Melhora no cognitivo, agora ele concentra mais e tem menos agressividade mais focado" (E10).

\section{Relação sociedade e família com o uso do CBD}

Alguns entrevistados relataram que compartilharam com a família e sociedade sobre o uso do CBD em seus filhos e tiveram boa aceitação em relação ao uso da substância no controle das crises epilépticas:

"Família supertranquila e disposta a sempre buscar o melhor. Sociedade pouco entende sobre o uso em crianças" (E2).

"Tanto a família quanto a sociedade apoiaram e acompanharam as dificuldades, anseios, desafios e vitórias no uso do CBD, sempre buscando esclarecer as dúvidas sobre as substâncias" (E8).

"A família aceitou a utilização da medicação, todos estão otimistas, a neurologista não se opôs a acompanhar, mas pediu atenção em relação ä confiabilidade do laboratório, e para eu ficar atenta para que a medicação não falte. Pois segundo ela, se a criança para de tomar ela pode entrar em estado de mal epiléptico" (E9).

"Pessoal ainda vê como uma alternativa e amenizar os sintomas da síndrome, mas não a cura (...)" (E10).

"Na minha família, foi tranquilo, são pessoas esclarecidas e de mente aberta" (E13).

Alguns depoentes relatam preconceito da família e/ou sociedade em relação ao uso da substância CBD nas crianças ou adolescentes:

"A minha família ainda é um pouco preconceituosa. Tanto que nem todos da minha família sabem do óleo... prefiro que não saibam para evitar de ouvir coisas desagradáveis e que me deixam tristes" (E6).

'Faz mais de dois anos que meu filho usa, a pouco tempo contei para alguns familiares e a maioria não sabe ainda do uso, por causa do preconceito e para evitar desgastes com explicações que acredito não serem necessárias" (E16).

\section{Entraves na aquisição do CBD}

Os pais e responsáveis relatam alguns desafios que eles enfrentaram na aquisição do CBD, como burocracia, alto custo da substância e das taxas de importação, desconhecimento médico, como dificultadores do processo:

"Excesso de burocracia, muitos documentos, valor muito alto" (E1).

"Burocracia, resistência e desconhecimento dos médicos" (E2). 
"Dificuldade de obter Importação" (E3).

“(...) depois que entramos com o processo de autorização na ANVISA, tínhamos que buscar o medicamento em Campinas, na alfândega, pagando mais taxas, hoje está mais tranquilo chega em casa pelos correios (...)" (E4).

"Primeiro achar um médico, depois conseguir a autorização pela Anvisa, por último conseguir importar e da continuidade pois além e ser muito caro a nossa justiça é muito falha, então é um desafio dar conta de continuar o tratamento sem ajuda do estado" (E5).

“(...) custo, desinformação, insegurança, processo judicial para aquisição e importação, neurologista sem conhecimento e com medo de prescrever uma substância desconhecida pela pesquisa Nacional, Anvisa libera ou não libera, demora na entrega, falta de informação dos bancos em realizar operações de pagamento de importação internacional e pôr fim a administração do CBD sem a certeza da dose exata e se realmente iria ou não dar certo (...). Além disso, neurologistas sem conhecimento e com medo de prescrever a substância (...)" (E8).

\section{DISCUSSÃO}

O CBD foi referido pelos pais e responsáveis das crianças e adolescentes como substância importante na redução das crises epilépticas. Segundo Blair RE, et al. (2015) os CBD têm sido usado medicinalmente há séculos, sendo que na última década, estudiosos tem explorado seu potencial terapêutico, particularmente no controle das crises epilépticas. Alguns estudos com CBD, tem demostrado atividade anticonvulsivante, enquanto em outros estudos experimentais sua eficácia para controle de crises ainda não foi totalmente estabelecida. Atualmente, há evidências preliminares de que os canabinóides não-psicoativos podem ser úteis como anticonvulsivantes, mas são necessários ensaios clínicos adicionais para avaliar a eficácia e segurança destes compostos para o tratamento da epilepsia.

No mesmo sentido, assim como pais e/ou responsáveis, Devinsky O, et al. (2017) relataram que estudo multicêntrico, duplo cego e randomizado, em pacientes de dois a oito anos com crises epilépticas com síndrome de Dravet evidenciou neste grupo uma redução significativa na convulsão tônica-clônica nos pacientes tratados com $20 \mathrm{mg} / \mathrm{kg} / \mathrm{dia}$ de CBD (Epidiolex®) quando comparados ao grupo placebo. O estudo mostrou uma redução das crises em $38,9 \%$ nos pacientes em uso do CBD versus $13,3 \%$ para o placebo $(P=$ 0,01). O número de pacientes com um decréscimo de $50 \%$ nas crises convulsivas também foi maior com o tratamento (43\% versus $27 \%$ placebo), embora a significância não foi atingida $(P=0,08)$.

Em outro estudo realizado por Devinsky $O$, et al. (2018) em pacientes de dois a 55 anos com síndrome Lennox-Gastaut, que apresentam crises epilépticas, resultou em diminuição significativa das crises epilépticas de $41,9 \%$ no grupo que utilizou o Epidiolex® a $20 \mathrm{mg} / \mathrm{Kg} / \mathrm{dia}$ e $37,2 \%$ no grupo que utilizou a $10 \mathrm{mg} / \mathrm{Kg} / \mathrm{dia}$, quando comparados com o grupo placebo que apresentou $17 \%$ de redução $(P=0,005$ e $P=$ 0,002 , respectivamente).

Thiele EA, et al. (2018) relatam em seu estudo duplo cego e randomizado, realizado em pacientes com Sindrome Lennox-Gastaut, que fizeram uso de Epidiolex® $20 \mathrm{mg} / \mathrm{Kg} / \mathrm{dia}$ comparados ao grupo placebo. No grupo do Epidiolex obtiveram redução significativa na mediana das crises epilépiticas (43,9\%) contra 21,8\% do placebo $(P=0,0135)$.

Borgan $\mathrm{F}$, et al. (2019) descrevem que o receptor canabinoides, modula o desempenho da memória através de mecanismos intracelulares e extracelulares que alteram a transmissão sináptica e a plasticidade. Embora a literatura tenha mostrado consistentemente que os usuários crônicos de cannabis apresentam acentuados prejuízos cognitivos, resultados mistos foram relatados no contexto de ensaios experimentais controlados por placebo. Portanto, não está claro se esses compostos alteram inerentemente os processos cognitivos ou se os indivíduos geneticamente predispostos a usar cannabis podem ter déficits cognitivos subjacentes. 
Tzadok M, et al. (2016), ressaltam que canabinoides como terapêutica raramente são usados sozinhos, estão como adjuntos de outras prescrições, como o caso da epilepsia. O uso de canabinoides como terapêutica adjuvante complica ainda mais a avaliação de sua eficácia para o tratamento da epilepsia no cenário clínico. Para estudar o uso de canabinoides para o tratamento da epilepsia, testes in vivo, podem fornecer informação, não só relacionada à sua eficácia, mas também o seu potencial.

Borgan $\mathrm{F}$, et al. (2019), descrevem que embora alguns pais tenham relatado que os medicamentos à base de cannabis tenham demonstrado eficácia nas crises epilépticas trazendo redução de crises, melhora cognitiva, diminuição de medicamentos alopáticos, controle mastigatório, melhora na qualidade de vida, melhora da coordenação motora, as evidências relativas aos seus efeitos cognitivos adversos ainda são pouco compreendidas.

A American Epilepsy Society (AES, 2019) descreve o uso do CBD como tratamento promissor com riscos e benefícios para crianças com epilepsia. Sugerem pesquisas com amostras maiores e com menos viés metodológicos. Os estudos atuais são o primeiro passo.

A maioria dos depoentes também apontam benefícios em relação à substância, apresentando melhora cognitiva, motora e melhor interação social com o uso do CBD e com a redução dos medicamentos anticonvulsivantes. Campbell CT, et al. (2017) descrevem que existem dois receptores canabinoides conhecidos, localizados no Sistema Nervoso Central (tipo I e II), sendo que o receptor tipo I melhora a memória, prazer, pensamento, concentração, percepções sensoriais e temporais. Mechoulam R, et al. (2002) encontraram em seu estudo, que crianças com epilepsia em uso de CBD mostraram melhorias na linguagem, habilidades motoras, comportamento e alerta, com efeitos adversos mínimos de sonolência e sintomas gastrointestinais.

Alguns pais e responsáveis relataram preconceito familiar e/ou da sociedade na utilização da substância. Blair RE, et al. (2015) relatam a importância da mídia na conscientização e compartilhamento de informações. Grupos de defesa, incluindo pais de crianças com epilepsia refratária que defendem tratamentos com CBD e estratégias opcionais adicionais, devem divulgar suas experiências junto aos meus de comunicação.

Elsohly M e Gul W (2014) descrevem que a sociedade ainda possui um certo preconceito em relação ao CBD pelo fato de ser um extrato da maconha, poucos sabem que a Cannabis pertence à família das plantas Cannabaceae, da qual existem três espécies principais que podem diferir em componentes bioquímicos: Cannabis sativa, Cannabis indica e a menos conhecida Cannabis Ruderalis (BARON EP, 2015). A planta de Cannabis contém mais de 200 compostos referidos como canabinoides.

Pelos resultados do estudo, percebeu-se vários pontos que os familiares descrevem como dificultadores, em relação à aquisição do $\mathrm{CBD}$ no Brasil, relacionados à prescrição médica, preço, importação da substância e desconhecimento médico. A Anvisa (2018) descreve o procedimento de importação do CBD, sendo necessário o laudo e a prescrição médica para o cadastramento do paciente na Anvisa e liberação da importação da substância.

Para o cadastramento, é necessário que o paciente envie o formulário de solicitação, declaração de responsabilidade ou laudo médico que justifique a solicitação. Após a análise do pedido, a Anvisa emite um parecer deferindo ou não a importação excepcional de produtos à base de CBD. Dessa forma, a compra se torna responsabilidade do paciente. Não cabe a Anvisa intervenção na escolha do produto solicitado pela equipe médica nem na sua forma de aquisição. Em 2015, a Anvisa regularizou no Brasil por meio da Resolução da Diretoria Colegiada (RDC) 17/2015, a importação de produtos à base de CBD e tetrahidrocannabinol (THC) (ANVISA, 2018).

Na Holanda diferentemente do Brasil, o óleo de CBD está disponível gratuitamente, porém sua composição e qualidade não são monitoradas sendo o óleo preparado por farmacêuticos mais caro e de difícil acesso. Problemas similares ocorrem no Brasil, onde os pais relataram dificuldade de acesso para aquisição e importação, valor elevado, dificuldade para obter prescrição, desinformação, dificuldade em acertar a dosagem do óleo sem a certeza se será realmente eficaz para cada criança (BARON EP, 2015). 


\section{CONSIDERAÇÕES FINAIS}

O estudo em questão mostrou que os pais ou responsáveis percebem o CBD como uma substância que pode reduzir os episódios de crise epiléptica em suas crianças ou adolescentes. Além disso, segundo os sujeitos entrevistados o CBD pode atuar na melhora cognitiva, motora e melhor interação social o que proporciona melhora na qualidade de vida. Relatam também, alguns entraves na aquisição da substância como alto custo, dificuldades na importação e desconhecimento médico. Desta forma faz-se importante o incentivo de estudos nessa área, para promover melhor, reduzir crises e ou abrandar reações geradas pelas mesmas, a fim de melhorar a qualidade dessas crianças e adolescentes e de seus responsáveis que necessitam enfrentar tanta burocracia no país para tratar seus filhos.

\section{REFERÊNCIAS}

1. AGÊNCIA NACIONAL DE VIGILÂNCIA SANITÁRIA (ANVISA). Orientações sobre importação de Canabidiol. 2018. Disponível em: http://portal.anvisa.gov.br/importacao-de- canabidiol. Acessado em: 04 de abr. de 2019

2. AMERICAN EPILEPSY SOCIETY (AES). 2019. American Epilepsy Society position statement on cannabis as a treatment for patients with epileptic seizures Disponível em: https://www.aesnet.org/about/positionstatements/american-epilepsy-society-position-on-cannabis-as-a-treatment-for-epileptic-seizures. Acessado em: 16 de jan. de 2022.

3. BARDIN L. Análise de conteúdo. São Paulo: Edições 70, 2011; 279 p.

4. BARON EP. Comprehensive review of medical marijuana, cannabinoids and therapeutic implications in medicine and headache: What a long and strange journey has been. Headache, 2015; 55(6): 885-916.

5. BLAIR RE, et al. Canabinoids: is there a potential role in the treatment of epilepsy?. Expert Opinion on Pharmacotherapy, 2015; 16(13): 1911-1914.

6. BORGAN F, et al. The effects of cannabinoid 1 receptor compounds on memory: a meta-analysis and systematic review across species. Psychopharmacology, 2019; 236(11): 3257-3270.

7. BRUCKI SM, et al. Cannabinoids in neurology - Brazilian Academy of Neurology. Arquivos de Neuro-Psiquiatria, 2015; 73(4): 371-374.

8. CAMPBELL CT, et al. Cannabinoids in pediatrics. Journal of Pediatric Pharmacology and Therapeutics, 2017; 22(3): 176-185

9. CONSELHO FEDERAL DE MEDICINA (CFM). Resolução CFM no 2.113/2014, de 16 de dezembro 2014.2014. Disponivel em: http://www.portalmedico.org.br/resolucoes/CFM/2014/2113_2014.pdf. Acessado em: 04 de abr. de 2019.

10. CONSELHO NACIONAL DE SAÚDE. Resolução no 466, de 12 de dezembro de 2012. 2012. Disponível em: https://conselho.saude.gov.br/resolucoes/2012/Reso466.pdf. Acessado em: 29 de jan. de 2022.

11. DEVINSKY O, et al. Effect of Cannabidiol on drop seizures in the Lennox-Gastaut Syndrome. The New England Journal of Medicine, 2018; 378(20): 1888-1897.

12. DEVINSKY O, et al. Trial of Cannabidiol for drug-resistant seizures in the Dravet Syndrome. The New England Journal of Medicine, 2017; 376(21): 2011-2020.

13. ELSOHLY MA, GUL W. Constituints de Canabis Sativa. In: PERTWEE R. Handbook of Canabis Sativa. Oxoford: Oxford University Press, 2014.

14. FONSECA GS, et al Epidemiological aspects of refractory epilepsy in a pediatrics Hospital Unit. Revista de Enfermagem UFPE on line, 2016; 10(supl. 3): 1466-1473.

15. GIL AC. Como elaborar projetos de pesquisa. 5aㅡ ed. São Paulo: Atlas, 2010.

16. GURGEL HLC et al. Uso terapêutico do canabidiol: a demanda judicial no estado de Pernambuco, Brasil. Saúde e Sociedade, 2019; 28(3): 283-295.

17. MECHOULAM R, et al. Cannabidiol: an overview of some pharmacological aspects. The Journal of Clinical Pharmacology, 2002; 42(S1):11S-19S.

18. MINAYO MCS. Pesquisa social: teoria, método e criatividade. 34. ed. Petrópolis, RJ: Vozes, 2015.

19. MINISTÉRIO DA SAÚDE. Avaliação e Conduta da Epilepsia na Atenção Básica e na Urgência e Emergência. $2018 a$. Disponível em: https://bvsms.saude.gov.br/bvs/publicacoes/avaliacao_conduta_epilepsia_atencao_basica.pdf. Acessado em: 29 de jan. de 2022.

20. MINISTÉRIO DA SAUDE. Secretaria de Atenção à Saúde. Portaria no 17, de 21 de junho de 2018. Aprova o Protocolo Clínico e Diretrizes Terapêuticas da Epilepsia. 2018b. Disponível em: http://conitec.gov.br/images/Protocolos/PCDT_Epilepsia.pdf. Acessado em: 08 de fev. de 2019.

21. OLIVEIRA MT, PAIM RSP. O uso terapêutico de canabinóides em pacientes portadores de doenças crônicas. 2015. Disponível em: https://www.semanticscholar.org/paper/O-USO-TERAP\%C3\%8AUTICO-DE-CANABIN\%C3\%93IDESEM-PACIENTES-DE-Oliveira-Paim/f7f915864669d8a9c91aa38eaee682667a84941c. Acessado em: 29 de jan. de 2022.

22. REDDY DS, GOLUB VM. The pharmacological basis of Cannabis therapy for epilepsy. The Journal of Pharmacology and Experimental Therapeutics, 2016; 357(1): 45-55.

23. REKTOR L, et al. Third international congress on epilepsy, brain and min: part 2. Epilepsy and Behavior, 2015: 138159.

24. THIELE EA, et al. Cannabidiol in patients with seizures associated with Lennox-Gastaut syndrome (GWPCARE4): A randomised, double-blind, placebo-controlled phase 3 trial. The Lancet, 2018; 391(10125): 1085-1096.

25. TZADOK M, et al. CBD-enriched medical cannabis for intractable pediatric epilepsy: The current Israeli experience. Seizure, 2016; 35: 41-44.

26. VINUTO JA. Amostragem em bola de neve na pesquisa qualitativa: um debate em aberto. Temáticas, 2014; 22(44): 203-220. 